\title{
Dialect Protection from the Perspective of Natural Characteristics Loss of Dialects
}

\author{
Chuqi Wang \\ Shandong University, Weihai Shandong, 264209, China
}

Keywords: Dialect, Natural characteristics, Dialect protection, Evolution.

\begin{abstract}
This paper analyzes the importance of dialect protection from the perspective of social and cultural construction in our country. It is found that in today's society, the dialect is on the verge of extinction, which has a serious impact on the inheritance and development of national folk culture, which has caused a serious blow on the diversity of native language in our country. Therefore, we must pay great attention to the development of dialect protection. This paper studies dialect protection from the perspective of natural characteristics loss of dialects in order to let more people recognize the importance and urgency of dialect protection and provide good support for the optimization of dialect protection.
\end{abstract}

\section{Introduction}

With the development of society and the integration of culture, language has been influenced by social progress and cultural development in the process of dynamic development for a long time. In the course of a long period of development, the laws and characteristics of features have formed, which have a significant impact on the inheritance and development of dialectal culture. During the course of five thousand years of developments in our country, a large number of dialects have been accumulated, and dialects have had an important influence on the diversity of our national culture. However, it should be noted that in today's society, natural characteristics loss of dialect is relatively serious, and it is imminent to optimize and innovate dialect protection. Therefore, it is very necessary to actively explore the optimization of dialect protection work in combination with the loss of the characteristic natural characteristics, and strives to create a favorable development environment for dialect protection.

\section{The evolutionary tendency of dialects and the resulting impact}

China has a vast territory and people in different regions are influenced by the regional characteristics and cultural exchanges during their development. Individual dialects have been formed. As a whole, there are many dialects, which is under a certain impact on the social and cultural construction. But with the development of the society in the new era and the frequent cultural exchanges, contacts between dialects and between dialects and mandarin gradually increased, and the depth of contacts deepened, which led to a certain degree of dialectal culture in different regions of our country. The dialect began to get closer to mandarin, which hindered the optimization of dialect protection [1]. A systematic analysis of the evolution of dialects shows that as cultural exchanges become more frequent, the development of dialects has also been affected, showing the basic development of a combination of autonomous evolution and passive evolution.

There is a certain inevitability in the development of society. The evolution of dialects is also influenced by the social progress, and the results of dialect evolution are often irreversible. The emergence of such situations will produce positive and negative effects on the development of dialects. On the one hand, it can enhance dialect development vitality and demonstrate the vitality of dialect development and inheritance,and on the other hand, it can be seen that in the process of dialect development, the natural characteristics are gradually disappearing and weakened, and the weakening of this primitive feature are focusing on the three aspects of speech, vocabulary and grammar, which 
leads to the gradual loss of its characteristics and serious impediments to the succession and development of dialects. Therefore, in the face of the reality of loss of natural characteristics in practice of dialect transmission, relevant researchers should correctly understand the importance of inheritance and protection of dialects and actively explore corresponding improvement measures to create favorable conditions for the protection and rescue of dialects as well as strive for the gradual settlement of the problem of the loss of natural characteristics, and comprehensively promote the optimization and protection of dialect protection and rescue work.

\section{The evolution and disappearance of the natural characteristics of the dialects in the transitional zone}

In the course of the development of dialect culture, the impact of the transitional zone is most obvious, and the evolution and disappearance of the natural characteristics of the dialects in the transitional zone are also relatively serious. Therefore, in the process of actual research, the evolution and disappearance of the natural characteristics of the dialects in the transitional zone are analyzed, and the dialect of Fei County is taken as the research object so as to gain an objective understanding on the evolution and disappearance of the natural characteristics of the dialects. We should deeply appreciate the urgency and necessity of dialect protection and rescue work and provide appropriate support for the overall optimization of dialect protection and rescue work.

\section{Dialect characteristics in the transitional zone}

The characteristics of the dialect in the transitional zone are concentrated in various aspects and exert some influence on dialect culture research. In the process of concrete analysis of the dialect characteristics of the transitional zone, we can make a detailed interpretation from the perspectives of central sounding and edge sounding. In other words, when a voice change, it is often influenced by geographical factors. It occurs at a certain geographical point first, and then begins to diffuse to the periphery from this point. During the diffusion process, the degree of variation is affected by the distance from the center point, such as the more distance, the sound power is more obvious, then change the characteristics is more neat, if the more distance, the sound power is more fuzzy, then the resulting change will be a serious lack of uniformity. Starting from this theory, we can see from the overall development of dialects in our country that not only the change of center tone and edge change will lead to the change of the dialect features in the transitional zone, but also the dialectal changes of authority in our society will cause profound impact, the lower the audience age group, the more serious it is affected by mandarin [2]. It can be seen from the historical development that with the passage of time, the succession groups of dialects in different age groups will weakened their grasp of the natural characteristic layer by layer, eventually resulting in the failure of dialect areas to guarantee the succession of dialects and seriously threaten the dialect evolution and development , which have a negative impact on the succession of dialect culture. Therefore, in the current social context, we should correctly understand the characteristics of the dialect in the transitional zone and the evolution of dialects, form an objective understanding of the dialect development process, and strive to explore dialect protection and rescue measures based on a comprehensive understanding and analysis of dialects, and laid a solid foundation for good cultural heritage.

Take Fei County dialect as an example to study the evolution of the natural characteristics Dialect of Fei County is a typical representative of the dialect of our country's transitional zone, and has formed a strong natural characteristic in the long-term development process. It is also highly valued in the inheritance and development of dialectal culture. In the research, we take Fei County dialect natural characteristics evolution as a research object, it has a certain reference value, which not only helps to form a detailed understanding of the evolution of dialect characteristics, but also helps the overall optimization of research work. From analysis of Study on Dialects of Shandong Province, this paper analyzes the dialect in Fei County, finds that because of the transitional zone, Fei County is relatively rich in linguistic system. Characteristics of dialects of Yinan and Mengdong in Dongying are relatively obvious in the north of Fei County, which should belong to Jilu Mandarin area. And 
even north of the region, such as Yishui area should belong to Jiaoliao Mandarin area. The Lanshan, Cangshan, Zaozhuang,including Fei County and other places belong to the Central Plains Mandarin area. So we can see that there is a special geographical location in Fei County, which is in the transitional zone. Fei County dialect also is affected to form obvious complex intersection characteristics. The special geographical location and special cultural exchange effect have added some value to the dialect study in Fei County, and also has a corresponding influence on the study of the nature of dialect in Fei County. In this study, we study and interpret the Nots on the dialect of Fei County, and find that, take the development of county dialect as the research object, the Fei County dialect system, involving a total of 24 consonants and 37 vowels, and a rich element Tone also. And we make an analysis on its tone, we find that they are mainly the High and Level, the Rising and the Falling Tones, transfer values were 213, 53, 55, 312. In addition, in the development process, dialect of Fei County formed a personalized character, there are special pronunciation phenomenon, that is,etc., in specific dialects, like the application of Rong[vən53], Shui, etc., and such application of initials has a certain relationship with the impact of Shanxi immigrants. Nowadays, the application of special initials has gradually been weakened in the inheritance and evolution of Fei County dialects, even in Fei County and the entire southwestern region, which have a negative impact on the succession and development of the Dialect in Fei County.

The analysis of the evolution of the natural characteristics in Fei County dialect is explained in detail, and the changes are mainly concentrated in the classification of the initial consonants of ancient Zhizhuang, Ancient fruit taken first-class opening and closing words such as the current vowels from the angle, reflecting the circumstances of the natural characteristic loss of dialect of Fei County in the evolution, which is thought-provoking. We took the phonetic variations of the Medieval mother alphabet for instance to make a more systematic analysis. The phonetic characters of this group of Chinese phonetic alphabet have some differences in the pronunciation of East-West dialect in Fei County. Eastern dialect usually reads zero-consonants in today's society, like Rang(Yang), Ren(yin), etc., Western dialects have obviously different characteristics, they cannot read zero-consonants. Compared with the pronunciation of Fei County, we found that the overall situation is essentially the same. Except for some local reading, others basically read 3 Tone[3]. And in this group, the words pronunciation can show distinctive characteristics belonging to The three day mother's voice, but this reading is common in older generations, subject to mandarin, in the young people, it has been basically read the zero-consonants, which shows the process of alternation between old and new, the natural characteristics of dialect are gradually weakened or disappeared.

\section{The urgency and necessity of dialect protection research}

We made a proper analysis on dialect evolution from the perspective of the natural characteristics loss of the dialects. We found that during the evolution, its diffuse development at the diachronic and synchronic level formed a certain degree of specificity, which has an important impact on the dialect cultural heritage [4]. Therefore, we made a detail analysis and interpretation of the development of dialects from the micro level. It is found that in the process of their evolution, the characteristics of dialects have undergone some adjustments and changes, which will eventually lead to serious consequences. In the dialect system, linguistic features with obvious inheritance characteristics and regional characteristics were gradually weakened, eroded and eventually disappeared in the dialect system, which seriously hindered the succession and development of dialect culture. At the same time, with the loss of the natural characteristics of obvious inheritance and regionalization in the evolution and diffusion of dialects, the individualized features of dialects began to be weaken, the proximity to the Central Plains Mandarin was significantly enhanced, and even the development of some dialects was severely affected by Mandarin influences. For example, the rapid disappearance of in Fei County dialect is one of the more typical examples. It clearly reflects the natural characteristics loss of dialect, which arouses great concern of researchers in modern society. It should be noted that in the process of dialect development, once there is a natural characteristic loss of dialect, the individuality of an individual dialect cannot be talked about. Even when we analyze in some sense, the natural characteristic of the dialect is essentially the vitality of dialect, while the loss of natural characteristic 
is the overdrawn to the vitality of the dialect system. Once the vitality is exhausted, the dialect will disappear in the long process of historical development, which has a serious negative impact on the construction of both the national culture and regional culture.

In view of this, in the background of new era, in the face of the reality of the loss of the natural characteristics of dialect, the research community in our country has begun to pay more attention to the protection and inheritance of dialects, and made the protection and inheritance of dialect a certain exploration, and strengthened the application of modern information technology, tried to build a dialect sound database, and achieved initial results, which provided good support for the overall promotion of dialect protection. It is because of recognizing the endangered dialect in the current situation that the natural characteristic of the dialect is lost. Dialect researchers realized the importance and urgency of dialect protection. And from the microscopic point of view to refine the analysis, the dialect types in our society are relatively more, individual dialect components of different types are reflected from different levels, and the special features of dialect, especially in some dialect with obvious geographical features in the system, the individual characteristics become more obvious. The loss of the natural characteristics is often influenced by complicating factors. Therefore, only by strengthening the research on the protection and rescue of dialects can we change the passive situation and form an active research situation and enhance the effectiveness of the research to provide an appropriate support and protection for the overall optimization of dialect protection and rescue work. It is worth mentioning that in the process of investigating the dialect protection and rescue efforts in an all-round way, although the dialect natural characteristic loss is relatively serious both in macro and micro perspectives, the dialect has not been absorbed completely, for the dialectal cultural system itself has shown some defensive characteristics, which has been analyzed from the perspective of dialect itself. The evolution and proliferation of dialectal cultural systems tend to be concentrated in a specific area. Therefore, under the contemporary social background, in the face of the loss of the natural characteristic of dialect, we can still discover the historical value and social value of dialect protection and rescue [5]. Therefore, relevant researchers in the new era must attach great importance to innovation and optimization of dialect protection and rescue work. While promoting dialect protection in an all-round way, they should strive to bring the role of the masses from different regions and through their identity of dialect culture into protection work, which achieves significant protection results and created favorable conditions for the succession and dissemination of our language and culture.

\section{Conclusion}

To sum up, judging from the loss of natural characteristics of dialects, the dialect should be analyzed and interpreted from the perspective of overall situation and long-term perspective and we should actively explore personalized protection programs based on the development of the times and the loss of natural characteristics of the dialect and strive to provide effective support for the optimization of dialect protection so as to create favorable conditions for the socialist spiritual and cultural construction in our country and earnestly speed up the process of socialist modernization and development.

\section{References}

[1] Zhang Weiiing. Dialect Protection from the Perspective of Dialect "Essence" Loss,Shandong Social Sciences, 2014 (4): 108-112.

[2] Feng Jiani. An analysis on the Relation between the Promotion of Mandarin and Dialect Protection, New Western (lately), 2016 (1): 83-82.

[3] Li Tian. Analysis on Dialect protection from the perspective of language attitude, Modern Lanquages (Academic Comprehensive), 2015 (5): 128-130.

[4] Xu Haitao. Analysis of the practical significance of dialect protection, Contemporary Educational Practice and Teaching and Research (Electronic Journal), 2017 (4): 525.

[5] Gao Kaige. A New Exploration of Dialect Protection under the Cultural Philosophy of Marxism, Brand, 2015 (4): 120-120. 